\title{
Comparative analysis of genetic diversity in the mangrove species Avicennia marina (Forsk.) Vierh. (Avicenniaceae) detected by AFLPs and SSRs
}

Received: 3 October 2000 / Accepted: 19 February 2001

\begin{abstract}
Avicennia marina is an important mangrove species with a wide geographical and climatic distribution which suggests that large amounts of genetic diversity are available for conservation and breeding programs. In this study we compare the informativeness of AFLPs and SSRs for assessing genetic diversity within and among individuals, populations and subspecies of A. marina in Australia. Our comparison utilized three SSR loci and three AFLP primer sets that were known to be polymorphic, and could be run in a single analysis on a capillary electrophoresis system, using differentcolored fluorescent dyes. A total of 120 individuals representing six populations and three subspecies were sampled. At the locus level, SSRs were considerably more variable than AFLPs, with a total of 52 alleles and an average heterozygosity of 0.78 . Average heterozygosity for AFLPs was 0.193, but all of the 918 bands scored were polymorphic. Thus, AFLPs were considerably more efficient at revealing polymorphic loci than SSRs despite lower average heterozygosities. SSRs detected more genetic differentiation between populations (19 vs 9\%) and subspecies (35 vs $11 \%$ ) than AFLPs. Principal co-ordinate analysis revealed congruent patterns of genetic relationships at the individual, population and subspecific levels for both data sets. Mantel testing confirmed congruence between AFLP and SSR genetic distances among, but not within, population comparisons, indicating that the markers were segregating inde-
\end{abstract}

Communicated by P. Langridge

T.L. Maguire

Department of Botany, The University of Queensland, Brisbane, QLD, 4072, Australia

R. Peakall (®)

School of Botany and Zoology, Australian National University,

Canberra, ACT, 0200, Australia

e-mail: rod.peakall@anu.edu.au

Tel.: +61-02-6125 0022, Fax: +61-02-6125 5573

P. Saenger

Centre for Coastal Management, Southern Cross University,

PO Box 157, Lismore, NSW, 2480, Australia pendently but that evolutionary groups (populations and subspecies) were similar. Three genetic criteria of importance for defining priorities for ex situ collections or in situ conservation programs (number of alleles, number of locally common alleles and number of private alleles) were correlated between the AFLP and SSR data sets. The congruence between AFLP and SSR data sets suggest that either method, or a combination, is applicable to expanded genetic studies of mangroves. The codominant nature of SSRs makes them ideal for further population-based investigations, such as mating-system analyses, for which the dominant AFLP markers are less well suited. AFLPs may be particularly useful for monitoring propagation programs and identifying duplicates within collections, since a single PCR assay can reveal many loci at once.

Keywords Avicennia marina - Mangrove · Microsatellite $\cdot$ SSR $\cdot$ AFLP $\cdot$ Genetic diversity

\section{Introduction}

Mangrove forests are a feature of the intertidal zone of the tropical and subtropical coastlines of the world. They comprise some 80 higher-plant species in several taxonomic groups. About half of these species occur in Australia, which is believed to be near one of the centres of mangrove evolution (Hutchings and Saenger 1987; Saenger 1998). Mangrove forests all over the world are heavily exploited for wood and fish-pond operations, as well as other activities. This exploitation of mangroves has resulted in the loss of valuable wood resources, that in turn may have led to losses of genetic diversity as well. To overcome these losses, mangrove reserves, afforestation schemes and enrichment plantings are now being established (Qureshi 1990; Saenger and Siddiqi 1993). In order to have a successful mangrove conservation program breeding program, or afforestation scheme, attention needs to be paid to the genetic structure within and between populations, and to the methods by which 
germplasm is collected and maintained. Because of the dynamic nature of the mangrove habitat, survival of mangrove seedlings is generally poor and replacementplanting often needs to be undertaken for up to 3 years (Saenger and Siddiqi 1993), adding to the demand for available germplasm. In addition, some mangrove species produce seeds which are only viable for a short time, leading to further difficulties in the collection and maintenance of germplasm.

Avicennia marina (Forsk.) Vierh., is an important mangrove species because it can grow and reproduce across a wide range of climatic edaphic, and tidal conditions. It is the most-widely distributed of all mangrove tree species, with an Indo-West Pacific distribution across the latitudes from $25^{\circ} \mathrm{N}$ to $38^{\circ} \mathrm{S}$. The structure of $A$. marina-stands varies from gnarled shrubbery on dry coastlines and coral atolls, to closed estuarine forests up to 40-m tall within larger systems of wet coastal tropical regions (Duke et al. 1998). The wide geographical and climatic distribution of A. marina indicates that there is a large amount of genetic diversity available, which can be exploited for conservation, breeding programs and afforestation schemes.

Among commercially important crop and tree species, two contrasting PCR-based techniques for assessing genetic diversity are now widely favoured over other PCRand non-PCR-based genetic marker methods: amplified fragment length polymorphisms (AFLPs) and microsatellites or simple sequence repeats (SSRs).

AFLPs are detected as dominant multilocus DNA fingerprints that are based on the selective PCR amplification of restriction fragments from total digests of genomic DNA. They combine the reliability of the RFLP technique with the power and ease of the PCR technique (Jones et al. 1997). Therefore, AFLPs are applicable to DNA of any origin or complexity without prior sequence information, primer synthesis, library construction or the characterisation of DNA probes (Zabeau and Vos 1993; Vos et al. 1995). As a consequence of these features, AFLPs are being used to investigate genetic variation in a growing number of microorganisms, plants and animals (Janssen et al. 1996; Karp et al. 1997; Mueller and Wolfenbarger 1999, O’Hanlon and Peakall 2000).

SSRs consist of tandemly repeated units of short nucleotide motifs, 1-6-bp long. These regions occur frequently and randomly throughout the genomes of plants and animals, and typically show extensive variation within and among individuals, populations and species (Jarne and Lagoda 1996). As multiallelic single-locus codominant genetic markers, both homozygotes and heterozygotes are detected, improving the ease of scoring and the information content per locus over AFLPs. This feature of SSRs combined with their hypervariability make them ideal for a wide range of applications in forensics, plant and animal breeding, conservation genetics, population genetics and molecular ecology (Chase et al. 1996; Jarne and Lagoda 1996; Karp et al. 1997; Goldstein and Schlötterer 1999). One drawback of SSRs is that library construction, DNA probing, DNA sequencing and primer synthesis is required to develop the
SSR assay. Fortunately, there is generally some crossspecies transferability of SSRs among closely related species that can help defray the initial development costs (Peakall et al. 1998; Maguire et al. 2000a).

Comparative studies of AFLP and SSR markers in a range of crop species, including soybean, maize, barley, wheat and coconut, have generally revealed good congruence between the genetic patterns revealed by the two genetic markers (Powell et al. 1996; Russell et al. 1997; Pejic et al. 1998; Donini et al. 2000; Teulat et al. 2000). One additional consistent and contrasting pattern has been observed. At the per-locus level, AFLP markers were considerably less variable than SSR loci, but on a PCR assay by assay basis the AFLP method consistently reveals many polymorphic loci per-PCR assay, compared with the usual single locus per-SSR assay (e.g. Powell et al. 1996). This difference in efficiency between AFLPs and SSRs can be partially offset by fluorescent-based multiplexing of SSR loci within PCR assays and/or gel lanes. Even so, with the potential to produce $10-100$ s of polymorphic loci per lane, the AFLP method may still surpass SSRs in efficiency. This efficiency, and the ease to which the technique can be applied to any organism will ensure that AFLPs remain a method of choice for a range of different studies (Mueller and Wolfenbarger 1999).

Maguire et al. (2000a) have previously described the characterisation and analysis of 16 SSR loci in A. marina and related species. Subsequently, the three most informative SSR loci were used to assess population genetic variation throughout the worldwide range of A. marina (Maguire et al. 2000b). Worldwide, a total of 70 alleles were detected at these three loci, but allelic diversity was not distributed equally across the species with populations at the extreme of the species distribution showing reduced heterozygosity and significant inbreeding. Overall, high levels of genetic differentiation were observed among-populations, with some $40 \%$ of the total variation representing among population differences. The finding of strong genetic structure refutes the hypothesis of widespread dispersal, despite the extensive distribution of the species.

To complement the SSR study of Maguire et al. (2000b) and given the efficiency promised by the AFLP technique, the aim of this study was to compare the patterns of genetic diversity in the mangrove A. marina as dected by AFLPs and SSRs. Since highly informative patterns of genetic variation were detected with just three SSR loci in the earlier study, we chose to compare the results at these loci with three AFLP primer combinations that were known a priori to be highly polymorphic. In this way, our comparison of the two methods involved an equivalent genotyping effort.

The specific objectives were to: (1) assess the levels of genetic diversity in A. marina within Australia at preselected AFLP and SSR loci, (2) to compare the informativeness of the chosen AFLP and and SSR loci at the level of individuals, populations and subspecies, and (3) to identify any implications of our findings for future mangrove conservation and breeding programs. 


\section{Materials and methods}

The study species and sampling

A. marina has wide variation in morphological characters, some of which appear to be influenced by ecological and environmental factors (Duke 1990). Three intraspecific groupings have been recognised based on morphological characters (Duke 1991; Everett 1994), although there remains some question as to whether these groupings truly represent subspecies or other intraspecific forms, or are simply variants influenced by environmental factors (Duke et al. 1998)

Within Australia, the subspecies australasica is clearly defined in distribution and morphology and is distinguished from the other two subspecies by having grey fissured and pustular bark, instead of the chalky green, smooth, and sometimes flaky bark of the others. The calyx also has narrow glabrous margins, whereas the other two subspecies have pronounced glabrous margins. The ratio of the width of the margin to the length of the calyx is less than 0.2 , in comparison to the other two subspecies, having a ratio greater than 0.2 (Duke et al. 1998). Differences between the subspecies eucalyptifolia and subspecies marina are, however, more subtle.

\section{Plant sampling and DNA isolation}

A total of 120 individuals of A. marina, representing six diverse natural populations, were sampled throughout its range in Australia. Leaf material from 20 randomly selected trees at each location were collected. The locations were as follows: (1) Darwin Harbour, Northern Territory (NT), (2) Moody Creek, Cairns, Queensland (QLD), (3) Ballina Reserve, New South Wales (NSW), (4) Bunbury Reserve, Western Australia (WA), (5) St Kilda Reserve, South Australia (SA), and (6) Port Albert, Victoria (VIC). All three subspecies were represented by this sampling. A. marina subspecies australasica, was represented by the SA, VIC and NSW populations. The subspecies eucalyptifolia was sampled in NT and QLD, while the subspecies marina was represented by a single population from WA.

Leaf material was stored at $-80^{\circ} \mathrm{C}$ for later analysis. Total genomic DNA was isolated from leaf tissue using a modified CTAB method (Maguire et al. 1994).

\section{Genetic analysis}

Primer sequences and PCR conditions for the three SSR loci, M3 $\left(\mathrm{TG}_{15}\right), \mathrm{M} 40\left(\mathrm{AG}_{32}\right)$ and M47 $\left(\mathrm{CA}_{13}\right)$, are given in Maguire et al. $(2000 \mathrm{a}, \mathrm{b})$. During PCR the three SSR loci were fluorescently labelled in different colors (FAM, TET, HEX), allowing them to be analysed simultaneously via capillary electrophoresis on a PE Applied Biosystems 310 Genetic Analyser as described by Maguire et al. (2000b).

For the purpose of this comparative study, we restricted the number of AFLP primer combinations to three, each labelled with a different color, such that the combined AFLP profiles could be run in a single analysis. In this way, three PCR reactions were required for each AFLP assay (following initial ligation and preselective amplification), matching the number of PCR reactions required for the SSR assay.

The fluorescent-based Perkin Elmer AFLP Plant Mapping Kit for regular plant genomes $(500-600 \mathrm{Mb})$ was used according to the manufacturer's instructions, with one exception. The reaction volume was halved for the selective amplifications, resulting in identical AFLP patterns and cost savings. This kit includes eight EcoRI and eight MseI primers, with a total of 64 primer combinations available. Initially, 21 primer combinations were examined for three individuals of A. marina, representing three distinct natural populations. All 21 primer combinations produced a large proportion of polymorphic bands, with relatively few monomorphic bands. The average number of bands detected per primer combina- tion ranged from 23.6 to 56.3 with a mean of 42.3 (size range 50 to $500 \mathrm{bp}$ ). From this pilot study, the three primer combinations that produced the most polymorphic bands were selected and applied to all 120 individuals of $A$. marina. The selected primers were EcoAAC/MseCAA, EcoAAG/MseCAA and EcoACA/ MseCAT, which were fluorescently labelled with TAMRA, JOE and FAM respectively (Perkin Elmer Applied Biosystems). The primers were also tested for reproducibility across different restriction-ligation digests of the same individuals, as well as preamplifications and selective amplifications. We found the AFLP technique to highly reproducible, both in peak height and position, as detected using the ABI 310 Genetic Analyser (data not shown).

\section{Data scoring, allele frequency and heterozygosity}

Following capillary electrophoresis, GeneScan and Genotyper software (PE Applied Biosystems) were used to aid the scoring of the SSR and AFLP profiles. The multilocus AFLP profiles were scored for band presence (1) or absence $(0)$ to create binary matrices. For the SSR loci, homozygous and heterozygous genotypes were inferred from the banding patterns and allele frequencies calculated directly from these genotypes. For the AFLP data, where only two states (present and absent) can be distinguished at each band position, it was assumed that each band corresponded to a locus with two alleles. Presence represents a dominant homozygote (AA) or heterozygote (Aa), and absence represents a recessive homozygote (aa). The unbiased estimator of Lynch and Milligan (1994) was used to estimate the allele frequency of p (A) and $\mathrm{q}$ (a). This estimator assumes independent nuclear loci and Hardy Weinberg equilibrium. The later assumption, is known to be violated in some populations of A. marina, including several populations sampled in this study, where some inbreeding appears to be occurring (Maguire et al. 2000b).

Mean expected heterozygosity values $(\mathrm{H})$ were calculated for both SSRs and AFLPs and multiplied by the number of loci analysed simultaneously (in each gel lane) to give the marker index (MI) as defined by Powell et al. (1996).

\section{Comparisons of genetic patterns}

In most studies that have compared codominant SSRs with dominant markers, such as RAPDs and AFLPs, the SSR data is simply coded as binary data so as to match the binary dominant data set(s). This approach for coding SSR data violates assumptions of independence among loci and results in a loss of information when genotypes are highly heterozygous. Fortunately, these concerns are likely to be minimised for studies of predominantly inbred lines (Powell et al. 1996), a condition that characterises most comparative studies to-date (eg. Powell et al. 1996; Russell et al. 1997). In contrast, mangroves are predominantly outbreeders and therefore require an alternative approach for coding the codominant data.

Peakall et al. (1995) introduced a method for calculating individual by individual genetic distances from codominant markers This in turn enables a common pathway for subsequent statistical analysis of both dominant and codominant data sets. For example, cluster analysis, correlation analysis by Mantel testing and an analysis of molecular variance (AMOVA) are based on these genetic distance matrices. All analyses were performed within GenAlEx V5 (Peakall and Smouse 2001) which provides a common pathway for the analysis of both dominant and codominant data sets.

Individual genetic distance metrics

The calculation of genetic distances for the AFLP data set followed the method of Huff et al. (1993) and Peakall et al. (1995) as:

$\mathrm{E}=\mathrm{n}[1-(2 \mathrm{nxy} / 2 \mathrm{n})]$, 
where $\mathrm{n}$ is the total number of polymorphic bands and $2 \mathrm{nxy}$ is the number of markers shared by two individuals. Both band-presence and band-absence are considered informative in this analysis. This is a true Euclidean metric as required for the subsequent analysis of molecular variance. Genetic distance matrices for each AFLP primer-set on their own, and the total data set (three primer sets combined) were calculated. In addition, a total genetic distance matrix was constructed based only on those bands that had a frequency of band presence $\geq 0.05$ in the total data set.

Pairwise individual by individual genetic distances for the codominant SSR data set were calculated following the method of Peakall et al. (1995) and further explained in Smouse and Peakall (1999). In brief, for a single-locus analysis of an SSR marker, with $i$-th, $j$-th, $k$-th and $l$-th different alleles, a set of squared distances is defined as $\mathrm{d}^{2}(i i, i i)=0, \mathrm{~d}^{2}(i j, i j)=0, \mathrm{~d}^{2}(i i, i j)=1, \mathrm{~d}^{2}(i j, i k)=1$, $\mathrm{d}^{2}(i j, k l)=2, \mathrm{~d}^{2}(i i, j k)=3$, and $\mathrm{d}^{2}(i i, j j)=4$. Genetic distance matrices for each SSR locus and the total, summed across loci under the assumption of independence, were calculated.

Analysis of molecular variance

For the AFLP and SSR data sets, the individual pairwise genetic distance matrices were analysed by the analysis of molecular variance (AMOVA) approach first developed by Excoffier et al. (1992) and extended to codominant data by Peakall et al. (1995) and Michalakis and Excoffier (1996). Total genetic variation was partitioned into three levels: among subspecific taxa, among populations within subspecies, and among individuals. Our nomenclature follows that of Peakall et al. (1995) with the exception that the term 'regions' is taken here to mean subspecies. Variation was summarized both as the proportion of the total variance and as $\phi$-Statistics or $F$-Statistic analogues; $\phi_{\mathrm{PR}}, \phi_{\mathrm{RT}}$ and $\phi_{\mathrm{PT}}$. $\phi_{\mathrm{PR}}$ represent the correlation between individuals within a population relative to that of individuals from the same subspecies, $\phi_{\mathrm{RT}}$ is the correlation of individuals from the same subspecies, relative to that of individuals from the species, and $\phi_{\mathrm{PT}}$ is the correlation between individuals within a population, relative to that of individuals from the species. The relationship between values of $\phi$ is given by:

$\left(1-\phi_{\mathrm{PT}}\right)=\left(1-\phi_{\mathrm{PR}}\right)\left(1-\phi_{\mathrm{RT}}\right)$, see Peakall et al. (1995).

Statistical significance was tested by random permutation, with the number of permutations set to 1000 .

Note that for the codominant SSR data it is also possible to partition variation within individuals (Peakall et al. 1995; Michalakis and Excoffier 1996), but this option was suppressed, since an equivalent analysis is not possible for the AFLP data set. In addition to the above calculations, pairwise $\phi_{\mathrm{PT}}$ values amongst all population pairs and among the three subspecies pairs were calculated.

Cluster analysis of individuals and populations

In order to more-effectively view the patterns of individual and population genetic distance, principal co-ordinates (PCO) analysis of both the individual genetic distance and population $\phi_{\mathrm{PT}}$ matrices for both the AFLP and SSR data sets were performed. Patterns revealed by the first two principle coordinate axes were found to be representative of higher order axes, and so only the first two dimensions were plotted in this paper.

\section{Correlation between AFLPs and SSRs}

The correlation between AFLP and SSR genetic distance matrices was investigated at three levels by the Mantel test of matrix correspondence (Mantel 1967; Smouse et al. 1986; Peakall et al. 1995): individual genetic distance, population $\phi_{\mathrm{PT}}$ distance and subspecific $\phi_{\mathrm{PT}}$ distances. Statistical significance was determined by random permutation, with the number of permutations set to 1000 for each test.

\section{Results}

Molecular marker variation

Within the 120 individuals of A. marina, a total of 52 SSR alleles were detected across the three polymorphic loci, with the number of alleles per locus ranging from 9 to 32 (mean=17.3). The inferred number of SSR repeats per locus ranged from 3 to 18 for locus M3, from 5 to 52 for locus M40, and from 5 to 15 for locus M47. The three AFLP primer sets detected a total of 918 bands, with the number of bands ranging from 298 to 319 (mean=306). No monomorphic bands were detected. Many of the AFLP bands were only encountered at low frequency, with 317 of the total bands (34.5\%) occurring at a frequency of less than 0.05 in the total data set. The remaining bands more or less evenly represented the full range of possible frequencies, with the exception that only three bands were found with a frequency $\geq 0.95$ (data not shown).

For SSRs, the expected heterozygosity $(\mathrm{H})$ for each locus was $0.715,0.904$ and 0.716 for M3, M40 and M47 respectively, with a mean of 0.778 and a marker index (MI) of 2.34. For the AFLPs, the average heterozygosity for each primer combination was $0.168,0.206$ and 0.207 for the primer sets EcoAAC/MseCAA, EcoACA/ $M s e \mathrm{CAT}$ and EcoAAG/MseCAA respectively. The average heterozygosity over the AFLP primer sets was 0.193 , and the marker index was 177 . Average heterozygosities for SSRs versus AFLPs were significantly different $(F=83.1, P<0.000, d f=5)$.

\section{Cluster analysis of individuals}

The results of PCO analysis of the pairwise individual genetic distance matrices are shown in Fig. 1 for AFLPs and SSRs. For the AFLPs, the first two axes only explain $15 \%$ of the variation; nevertheless, distinct clusters are apparent. With one exception, all samples from subspecies marina (W) from Western Australia form a distinct group. Individuals representing subspecies australasica (S) from SA form a distinct cluster adjacent to the intermixed group of samples of the same subspecies from NSW (N) and Victoria (V). Sample of subspecies eucalyptifolia from Qld (Q) and NT (T) are partially intermixed, but show little overlap with the other subspecies.

The first two axes of the PCO analysis for the individual SSR distance matrix explain $42 \%$ of the variation (Fig. 1). Yet in contrast to the AFLP results, the clusters are less discrete (reflecting greater variation amongst the individuals) although the general patterns are similar. With one exception, individuals of the subspecies marina from WA are distinct. On the other hand, the placement of South Australian (S) individuals of subspecies australasica range from close to individuals of subspecies marina to intermixed with NSW (N) and Victorian (V) samples of australasica. As for the AFLPs, the individuals of subspecies eucalyptifolia from Queensland (Q) and the Northern 


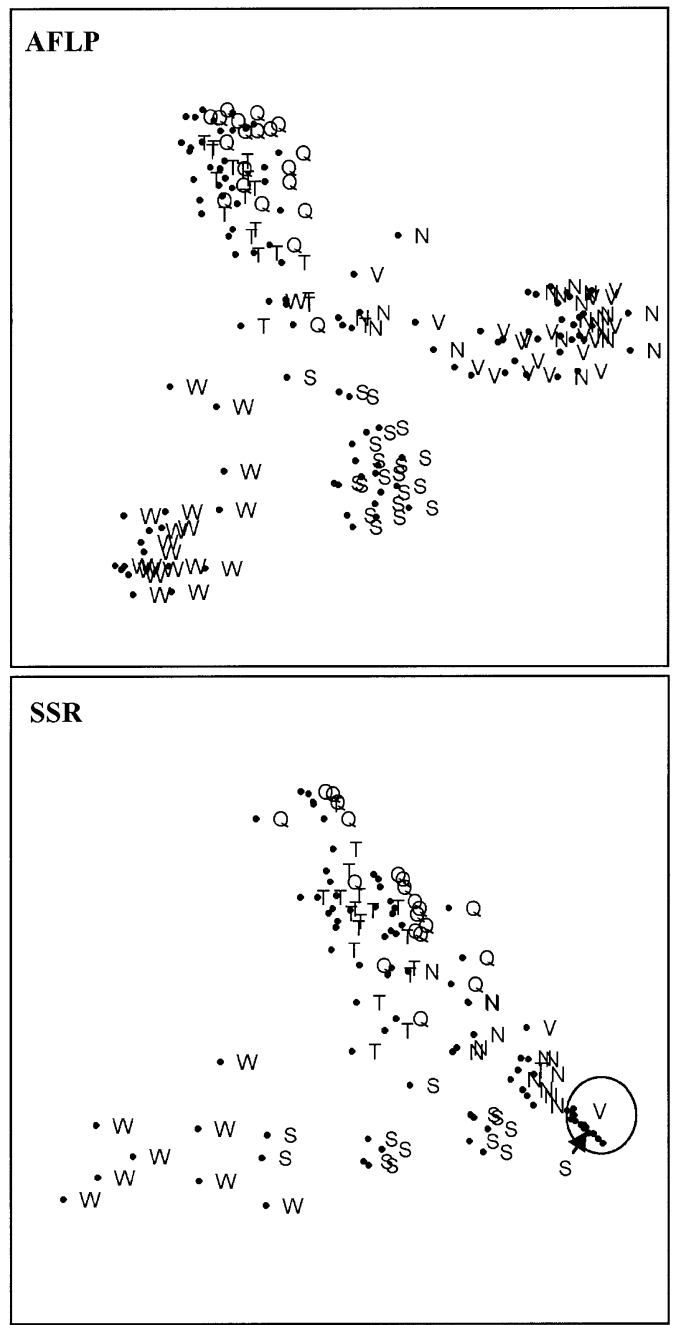

Fig. 1 PCO plots of the first two axes based on the individual AFLP and SSR distance matrices. The circle encloses a cluster of individuals from Victoria and one individual from South Australia. Populations are coded as $(N)$ New South Wales, $(Q)$ Queensland, $(S)$ South Australia, $(T)$ Northern Territory, $(V)$ Victoria, $(W)$ Western Australia
Territory (T) were intermixed and largely distinct from the subspecies australasica.

\section{Analysis of molecular variance}

Figure 2 summarizes the results of analysis of molecular variance for the total AFLP and SSR data sets. For AFLPs, $11 \%$ of the genetic variance was accounted for by variation among the subspecies, with a further $9 \%$ accounting for variation among populations within subspecies, with the remainder (80\%) represented among individuals within populations. By contrast, for SSRs, 35\% of the total genetic variance represented subspecies differences and a further 19\% variance among populations within subspecies, with only $46 \%$ of the total variance
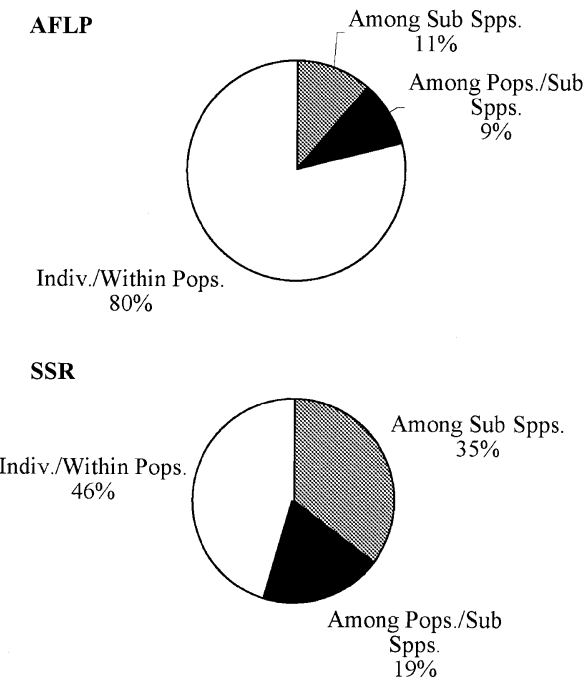

Fig. 2 Results of AMOVA for the total AFLP and SSR data sets showing the percentage of variation apportioned among subspecies, populations and individuals
Table 1 Results of AMOVA for AFLP and SSR data sets showing the percentage of variation apportioned among subspecies, populations and individuals for individual AFLP primer sets, individual SSR loci and the total

a Primer set EcoAAC/MseCAA b Primer set EcoAAG/MseCAA ${ }^{c}$ Primer set EcoACA/MseCAT

\begin{tabular}{lccccc}
\hline AFLPs & Total & P1 $^{\mathrm{a}}$ & $\mathbf{P 2}^{\mathrm{b}}$ & $\mathbf{P 3}^{\mathrm{c}}$ & Freq $>\mathbf{0 . 0 5}$ \\
\hline$n$ Bands & 918 & 298 & 301 & 319 & 601 \\
& $\%$ & $\%$ & $\%$ & $\%$ & $\%$ \\
Among subspecies & 11 & 13 & 9 & 12 & 12 \\
Among pops./subspecies & 9 & 8 & 11 & 9 & 10 \\
Indiv./within Pops. & 80 & 79 & 80 & 79 & 78 \\
$\phi_{\mathrm{RT}}$ & 0.114 & 0.133 & 0.089 & 0.122 & 0.120 \\
$\phi_{\text {PR }}$ & 0.106 & 0.092 & 0.117 & 0.105 & 0.111 \\
$\phi_{\text {PT }}$ & 0.208 & 0.213 & 0.196 & 0.214 & 0.217 \\
\hline SSRs & Total & $\mathbf{M 3}$ & $\mathbf{M 4 0}$ & $\mathbf{M 4 7}$ & \\
\hline$n$ Alleles & 52 & 11 & 32 & 9 & \\
Among subspecies & $\%$ & $\%$ & $\%$ & $\%$ & \\
Among pops./subspecies & 35 & 45 & 12 & 50 & \\
Indiv./within Pops. & 46 & 14 & 27 & 36 & \\
$\phi_{\text {RT }}$ & 46 & 41 & 61 & 0.497 & \\
$\phi_{\text {PR }}$ & 0.353 & 0.449 & 0.119 & 0.322 & \\
$\phi_{\text {PT }}$ & 0.301 & 0.262 & 0.311 & 0.659 & \\
\hline
\end{tabular}


Table 2 Outcomes of Mantel tests for correlation $(r)$ between AFLPs and SSRs at the individual level for populations, subspecies and the total, and the probability of statistical significance $(P)$ based on the outcomes of 1000 random permutations

\begin{tabular}{lrl}
\hline Item & \multicolumn{1}{l}{$\boldsymbol{P}$} \\
\hline Population & & \\
SA & -0.060 & 0.665 \\
NSW & 0.092 & 0.147 \\
VIC & 0.116 & 0.160 \\
QLD & 0.255 & 0.027 \\
NT & 0.050 & 0.363 \\
WA & -0.062 & 0.627 \\
Subspecies & & \\
australasica & 0.207 & 0.013 \\
eucalyptifolia & 0.152 & 0.023 \\
marina & -0.062 & 0.627 \\
Total & & \\
All Pops & & 0.001 \\
\hline
\end{tabular}

among individuals within populations. Thus, the AFLPs detected less subspecies and population differentiation than the SSRs.

Table 1 summarizes the outcomes of the analysis of molecular variance for each AFLP primer set and for each SSR locus. There was little difference among the three AFLP primer sets in the distribution of genetic variance, and the results for the subset of bands with a frequency $\geq 5 \%$ was similar to the total data set. Thus, while 317 of the 918 (35\%) banding sites where characterized by band presence at $5 \%$ or less frequency, the inclusion of these low-frequency bands in the data set has little impact on the final results (Table 2). Greater variation was detected amongst SSR loci compared with the AFLPs. Most notable, were the results for the M40 locus. This locus exhibited approximately 3-times as many alleles as each of the other SSR loci and detected considerably less genetic variation among the subspecies $(12 \%)$ and more variance among the populations within subspecies $(27 \%)$ than the other two loci.

At the population level, pairwise $\phi_{\mathrm{PT}}$ values, while of different magnitudes, revealed congruent patterns of relationship between AFLPs and SSRs (Fig. 3). With both AFLPs and SSRs, the Northern Territory and Queensland populations of eucalyptifolia clustered together, while the Western Australian population of marina was an outlier. Within australasica there was some variation in the placement of three populations between AFLPs and SSRs, but all three populations representing this taxon formed a group.

\section{Correlations between AFLPs and SSRs}

A significant and positive correlation $(r=0.381, P<0.001)$ was found between linear AFLP and SSR individual pairwise genetic distance matrices (Fig. 4, Table 2). However, this positive correlation is somewhat mislead-

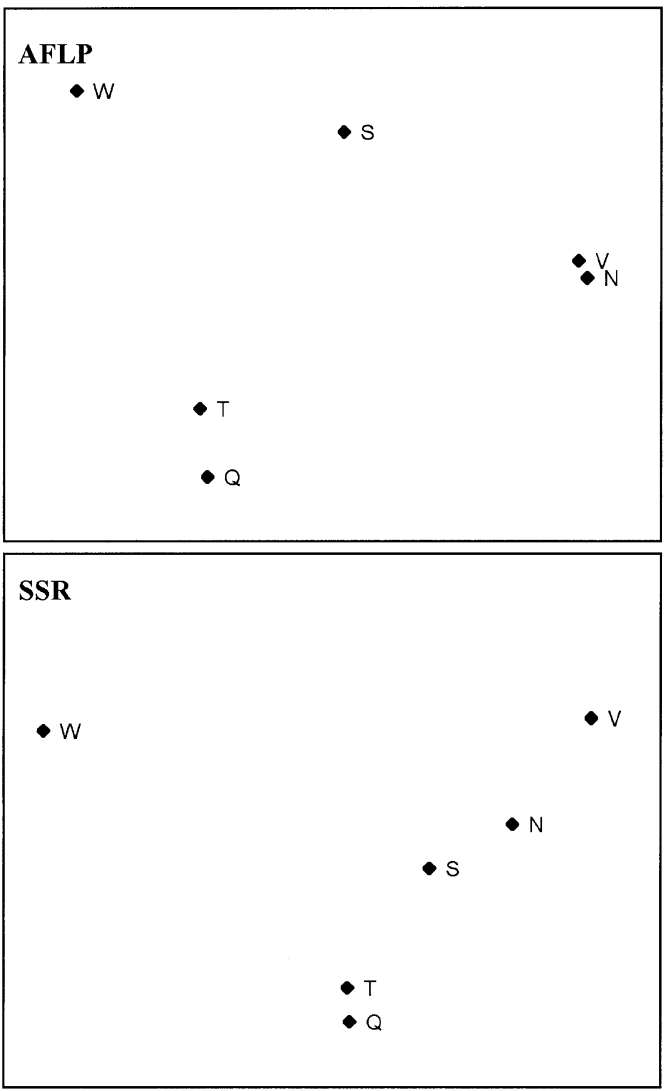

Fig. 3 PCO plots of the first two axes based on the population $\left(\phi_{\mathrm{PT}}\right)$ AFLP and SSR distance matrices. Populations are coded as $(N)$ New South Wales, $(Q)$ Queensland, $(S)$ South Australia, $(T)$ Northern Territory, $(V)$ Victoria, $(W)$ Western Australia
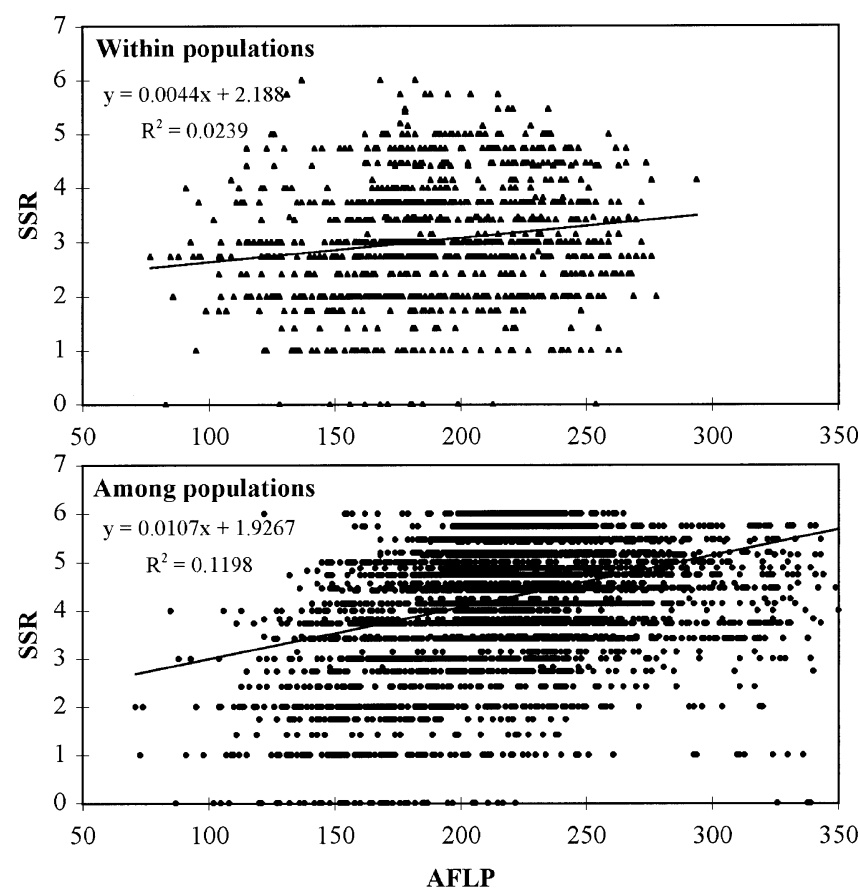

Fig. 4 Comparisons of the inter-individual AFLP and SSR genetic distances for within and among population comparisons 
Table 3 Outcomes of Mantel tests for correlation $(r)$ between AFLP primer sets and between SSR loci at the individual and population level, and the probability of statistical significance $(P)$ based on the outcomes of 1000 random permutations

a Primer set $E c o A A C / M s e C A A$ b Primer set EcoAAG/MseCAA c Primer set EcoACA/MseCAT

\begin{tabular}{|c|c|c|c|c|}
\hline & \multicolumn{2}{|c|}{ Individual level } & \multicolumn{2}{|c|}{ Population level } \\
\hline & $r$ & $\boldsymbol{P}$ & $r$ & $\boldsymbol{P}$ \\
\hline \multicolumn{5}{|l|}{ AFLPs } \\
\hline $\begin{array}{l}\mathrm{P} 1^{\mathrm{a}} \text { vs } \mathrm{P} 2^{\mathrm{b}} \\
\mathrm{P} 2 \text { vs P3c } \\
\mathrm{P} 2 \text { vs } \mathrm{P} 3\end{array}$ & $\begin{array}{l}0.677 \\
0.752 \\
0.698\end{array}$ & $\begin{array}{l}0.001 \\
0.001 \\
0.001\end{array}$ & $\begin{array}{l}0.857 \\
0.945 \\
0.871\end{array}$ & $\begin{array}{l}0.001 \\
0.001 \\
0.001\end{array}$ \\
\hline \multicolumn{5}{|l|}{ SSRs } \\
\hline $\begin{array}{l}\text { M3 vs M40 } \\
\text { M3 vs M47 } \\
\text { M40 vs M47 }\end{array}$ & $\begin{array}{l}0.198 \\
0.305 \\
0.179\end{array}$ & $\begin{array}{l}0.001 \\
0.001 \\
0.001\end{array}$ & $\begin{array}{l}0.463 \\
0.603 \\
0.567\end{array}$ & $\begin{array}{l}0.114 \\
0.054 \\
0.063\end{array}$ \\
\hline
\end{tabular}

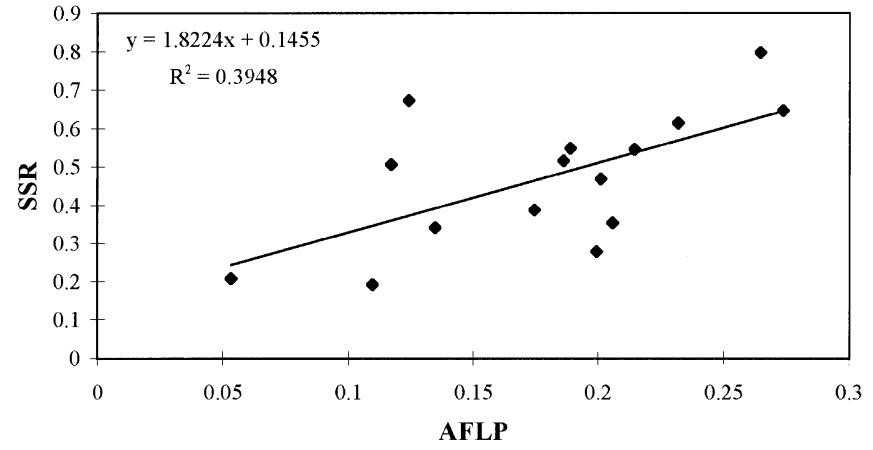

Fig. 5 Comparisons of the interpopulation $\left(\phi_{\mathrm{PT}}\right)$ AFLP and SSR genetic distances

ing. The within versus among population comparisons are graphed separately in Fig. 4 where it can be seen that any positive correlation in the total data set is largely due to the among population components. Further subdivision of the data set also shows this positive relationship between AFLPs and SSRs is largely absent at the population level (with the exception of Queensland), and weaker at the subspecies level (Table 2). This suggests that, at the individual level, there is little or no meaningful correlation between AFLP and SSR diversity as assessed by the loci used in this study.

Population $\phi_{\mathrm{PT}}$ distances showed much stronger correlation between AFLPs and SSRs $(r=0.628, P<0.002$, Fig. 5), than for the individual by individual correlations. The correlation between $\phi_{\mathrm{PT}}$ distances at the subspecies levels was also high $(r=0.998, P<0.137)$ although the small number of data points prevent a meaningful test of significance.

Collectively, the results from Mantel tests and comparisons of PCO plots at the population level (Fig. 5) suggest that while individual by individual correlations are poor or non existent, both the AFLP and SSR loci used here are detecting congruent patterns of genetic diversity at the population and subspecific levels.

\section{Correlations between AFLP primer sets and SSR loci}

Each AFLP primer set produced a large number of polymorphic bands. To test if the genetic patterns detected by one AFLP primer set were congruent with the patterns detected by another primer set, Mantel tests were performed at both the individual and population levels. The results are shown in Table 3. For each of the AFLP primer sets, a high and significant correlation was observed at the individual levels ( $r=0.67$ to 0.74 ), with even higher correlations at the population levels ( $r=0.86$ to 0.95 ). By contrast, for the SSR loci, the correlations between loci were much lower, but significant ( $r=0.18$ to 0.30$)$ at the individual level; with higher $(r=0.46$ to 0.60$)$, but nonsignificant values at the population level. Thus, the genetic patterns at a single SSR locus are poorly predicted at other SSR loci, although over sufficient loci we might expect stronger correlations to emerge, at least at the population levels.

\section{Discussion}

Polymorphism and marker efficiency

In this study we have assessed the patterns of genetic diversity within and among Australian populations of A. marina based on pre-selected sets of three SSR loci and three AFLP primer combinations. Although the genotyping effort, as defined by the number of PCR reactions and number of lanes required, was equivalent between the methods, many fold more loci were obtained by the multilocus AFLP. At the locus level, however, the multiallelic SSR loci were considerably more variable than the biallelic AFLP loci. The restriction in this study to a small set of pre-selected SSR loci may introduce bias in our comparison of AFLPs and SSRs, and therefore some caution is required when generalising beyond these specific loci. Nevertheless, while as yet there appear to be no other comparative studies of AFLP and SSR diversity at the individual and population levels within natural populations, our finding are matched by other comparative studies of these two genetic markers in agricultural plant systems. This suggests that our findings may well be general.

For example, the level of polymorphism detected by the mangrove SSRs and AFLPs was significantly different, with the SSR loci showing higher levels of heterozy- 
gosity than the AFLP loci. This is also a consistent finding of other studies (e.g. Powell et al. 1996; Russell et al. 1997; Pejic et al. 1998; Teulat et al. 2000), and probably reflects two major differences between the genetic markers. First, as typically dominant markers, AFLPs ordinarily only detect two alleles per locus, which reduces the maximum levels of heterozygosity possible to 0.5. Second, SSR regions are well known to exhibit much higher levels of mutation than other parts of the genome (Jarne and Lagoda 1996; Goldstein and Schlötterer 1999). The more-common genomic regions with lower mutation rates than SSRs, are likely to be frequently targeted by the AFLP method.

The overall utility of a given marker system is a balance between the level of polymorphism detected and the extent to which an assay can identify multiple polymorphisms. Despite lower average levels of polymorphism per locus, the AFLP method was the mostefficient marker, with over 900 polymorphic loci detectable in a single gel lane, compared to three SSR loci with 52 alleles. As a consequence the marker index (MI) was almost 100-fold higher for AFLPs (177) than SSRs (2.3).

While there is scope to expand the number of SSR loci that can be multiplexed in a single lane, AFLPs typically show a high marker index in comparison to SSRs (Powell et al. 1996; Russell et al. 1997; Pejic et al. 1998; Bohn et al. 1999). The multiplex ratio of AFLPs can be further adjusted by altering the restriction enzymes and the degree of $3^{\prime}$ nucleotide extension on the PCR primers; which offers a high degree of flexibility to the experimenter (Zabeau and Vos 1993).

Population and subspecific structure

At both the AFLP and SSR loci significant genetic structure was found between populations and subspecies. However, the degree of genetic differentiation differed between the markers. For AFLPs, $80 \%$ of the total genetic variation was accounted for by variation among individuals within populations, with the remaining variance split among subspecies $(11 \%)$ and populations (9\%). By contrast, across the three SSR loci there was considerably more genetic differentiation among the subspecies (35\%) and populations (19\%), with less than $50 \%$ of the variance distributed among individuals within populations (Fig. 2).

Thus, while both markers revealed significant differences at the population and subspecific levels, the degree of difference varied. Peakall et al. (1995) found a similar pattern when comparing population and regional differences in Buffalograss with allozymes and RAPDs. In this case, the most-variable genetic marker (RAPDs) showed the highest degree of population and regional differentiation. This may well reflect a consistent pattern, in which the most-variable marker system will show the most differentiation.

\section{Congruence of taxonomic relationship}

In general the PCO analysis for both individuals and populations (Figs. 2 and 3) were congruent between the AFLP and SSR loci. The subspecies marina represented by the population from WA was clearly distinct in all analyses. The two populations, NT and QLD representing the subspecies eucalyptifolia, cluster together and separately from the other subspecies. Representatives of australasica from NSW and VIC were also grouped together. However, in both individual AFLP and SSR analyses, individuals from the SA population of australasica were intermediate between australasica from NSW and VIC and marina from WA. Close inspection of the individual Principal Coordinate plot for SSRs (Fig. 1) further reveals that the placement of individuals varied from within the WA cluster to within the NSW/VIC cluster. Geographically, SA is located between the VIC and WA populations; thus, this population may share genetic characteristics from both subspecies and perhaps suggests that continuous variation occurs between the subspecies. Clearly more individuals and populations need to be sampled to clarify the taxonomic relationship of this population in SA. Expanding the number of SSR loci used may also aid in the clarification of relationships.

Broad congruence between the taxonomic patterns revealed by AFLPs and SSRs has been found in other comparative studies. However, the genetic relationships revealed by AFLPs correlated better with known pedigree relationships in barley (Russell et al. 1997) and maize (Pejic et al. 1998) than the relationships revealed by SSRs. Similarly, AFLPs showed a better resolution of genetic relationships than SSRs in coconut (Teulat et al. 2000). This finding in several comparative studies of crop species has led to the conclusion that SSRs may be less suitable for defining relationships than other markers (Russell et al. 1997; Teulat et al. 2000).

Several features of SSRs may contribute to a lower correlation between patterns of relationship than other methods. These include constraints on allele size, and rapid and complex mutational processes within the SSR and flanking regions that result in size homoplasy (Peakall et al. 1998; Fisher et al. 2000). Both these factors are likely to lead to underestimates of genetic diversity between more-distantly related taxa, which may limit the application of SSRs to defining relationships at lower taxonomic levels than for other markers (Peakall et al. 1998). The high levels of genetic diversity revealed by SSRs may further contribute to the lack of correlation, since genetic similarities will be typically lower with SSRs than other markers (Powell et al. 1996; Russell et al. 1997), potentially reducing the statistical power to define relationships. However, these factors are likely to be minimised by increasing the number of SSR loci used. In most studies to-date, a smaller number of SSR loci are used compared with many more loci from multilocus systems such as AFLPs. 
Congruence at the individual level

Despite strong concordance between AFLP and SSR loci at the population and subspecies levels, there was little concordance of inter-individual AFLP and SSR genetic distances in mangroves (Fig. 4). On close inspection, it is apparent that any correlation for the total data set reflects only a positive correlation between comparisons among the populations and subspecies, since within subspecies, and populations there was no concordance between SSR and AFLP diversity (Table 3). This lack of congruence may in part reflect the small number of SSR loci included in this study. However, a similar lack of inter-individual correlation between genetic markers was reported by Powell et al. (1996) within soybean. Genetic similarities based on 36 SSR loci showed no correlation with AFLP, RAPD and RFLP genetic similarities within Glycine max and Glycine soja comparisons, although pairwise comparisons between $G$. max and G. soja were correlated (Powell et al. 1996). This parallels our findings in mangroves in which there is a correlation only between more-distantly related comparisons. Interestingly, a similar pattern of correlation was found when allozymes and RAPD patterns were compared in buffalograss (Peakall et al. 1995). This may suggest that, in general, different marker systems are segregating independently, but the ecologically and evolutionarily important groups are similar between different markers (Peakall et al. 1995).

Implications for mangrove conservation and breeding programs

Knowledge of the genetic variation and genetic relationships between individuals and populations can be an important consideration for the efficient rationalisation and utilisation of mangrove germplasm resources. The genetic goal of both ex situ germplasm conservation and in situ population conservation is to maximise the protection of genetic diversity as efficiently as possible (Schoen and Brown 1993). Several different (but related) genetic criteria have been used to identify both germplasm accessions and natural populations of high conservation value. Allelic richness, or the number of alleles per locus, was first proposed by Marshall and Brown (1975). This criterion has since been widely used in many ex situ germplasm programs (Brown and Briggs 1991; Schoen and Brown 1993) and also to identify natural populations for conservation (Petit et al. 1998). Marshall and Brown (1975) further proposed that the most-important alleles to target in any sampling strategy are 'locally common alleles.' That is, alleles that are common in one to several populations, but not the species as a whole. While less-commonly used as a genetic criterion the number of rare, or unique alleles, has sometimes been advocated (Brown and Briggs 1991; Falk 1991). This criterion assumes rare alleles maybe evolutionarily important, but requires equivalent and intense
No. Alleles

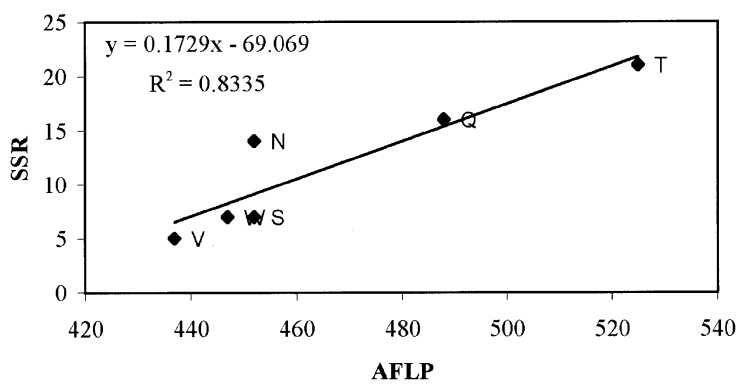

No. Locally Common Alleles

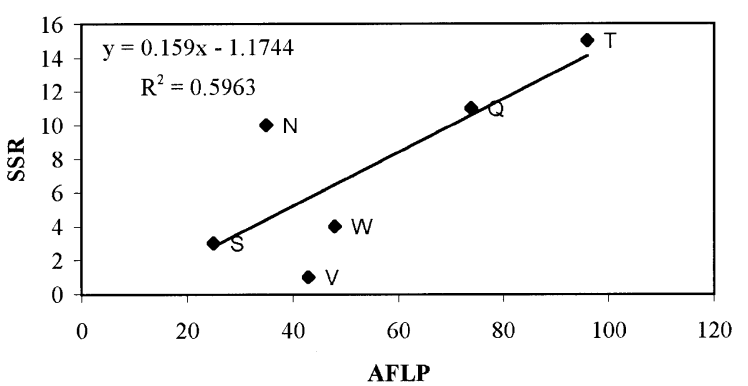

No. Private Alleles

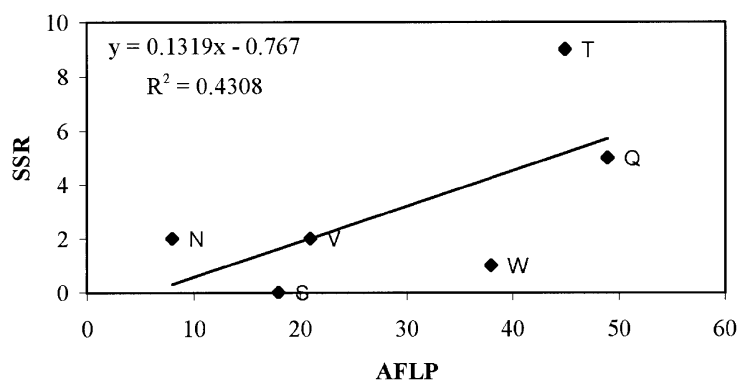

Fig. 6 Relationships between AFLP and SSR data sets for the number of alleles, number of locally common alleles and number of private alleles. Populations are coded as $(N)$ New South Wales, $(Q)$ Queensland, $(S)$ South Australia, $(T)$ Northern Territory, $(V)$ Victoria, $(W)$ Western Australia

genetic sampling amongst populations to ensure that the rare alleles are adequately sampled (Falk 1991).

In Fig. 6 we show the correlation between the AFLP and SSR loci investigated in A. marina for these three genetic criteria. A strong significant positive relationship between the number of AFLP bands per populations and the number of SSR alleles $\left(R^{2}=0.83, F=20.02, P=0.01\right.$ $d f=5$ ) was found. The NT population showed the highest number of alleles, and the VIC population the least, for both AFLPs and SSRs. A positive and significant relationship still holds if AFLP bands with a frequency $<0.05$ are excluded $\left(R^{2}=0.75, F=11.91, P=0.02 d f=5\right)$. There is also a positive relationship between the number of locally common alleles $\left(R^{2}=0.59, F=5.9, P=0.07\right.$ $d f=5$ ), defined here as AFLP bands or SSR alleles with frequency $\geq 0.05$ and restricted to one or two populations. Finally, although not significant, there is a trend 
towards a positive relationship for the number of private alleles $\left(R^{2}=0.45, F=3.03, P=0.15 d f=5\right)$, defined here as AFLP bands or SSR alleles restricted to a single population. Based on any one of these criteria, for either the AFLP or SSR data sets, the same priority populations for germplasm collections or in situ protection are identified. Clearly, within the subspecies eucalyptifolia the NT population is the most-genetically diverse, while within australasica the NSW population contains the most genetic diversity when evaluated across the three criteria.

Despite the small number of SSR loci used in this study, the general congruence between the AFLP and SSR data sets and their broad agreement with the morphological groups suggest that either method, or a combination of both, are applicable to expanded genetic studies of mangroves. Nevertheless, there are different strengths and limitations of the two systems, and knowledge of these may be used to guide the choice of marker system for a given question. For example, in this study the SSRs revealed considerably greater differences among populations and subspecies than the AFLPs; consequently, they may represent the best system for developing assays to quickly assign unknown individuals to populations or subspecies. The codominant nature of SSRs also makes them ideal for further population-based investigations, such as mating-system analyses, for which the dominant AFLP markers are less well-suited. On the other hand, AFLPs may be more useful for defining taxonomic relationships amongst mangrove species than SSRs. AFLPs may also prove useful for monitoring propagation programs and for identifying duplicates within collections, since a single PCR assay can reveal many loci at once.

Acknowledgements We thank Peter Smouse for his generous statistical advice, and The Centre for Plant Conservation Genetics, Southern Cross University, for laboratory space and invaluable discussion. We also thank the following people and organisations who kindly collected leaves of A. marina: E. Maguire, R. Harrison, Darwin Botanic Gardens, Department of Primary Industries Queensland, Department of Conservation and Land Management Western Australia. The Australian Centre for International Agricultural Research (ACIAR) is gratefully acknowledged for funding this investigation.

\section{References}

Bohn M, Utz HF, Melchinger AE (1999) Genetic similarities among winter wheat cultivars determined on the basis of RFLPs, AFLPs, and SSRs and their use for predicting progeny variance. Crop Sci 39:228-237

Brown AHD, Briggs JD (1991) Sampling strategies for genetic variation in ex situ collections of endangered plant species. In: Falk DA; Holsinger KE (eds) Genetics and conservation of rare plants. Oxford University Press, New York, pp 99-122

Chase M, Kesseli R, Bawa K (1996) Microsatellite markers for population and conservation genetics of tropical trees. Am J Bot 83:51-57

Donini P, Law JR, Koebner RMD, Reeves JC, Cooke RJ (2000) Temporal trends in the diversity of UK wheat. Theor Appl Genet 100:912-917
Duke NC (1990) Morphological variation in the mangrove genus Avicennia in Australasia: systematic and ecological considerations. Austr Systematic Bot 3:221-239

Duke NC (1991) A systematic revision of the mangrove genus Avicennia (Avicenniaceae) in Australasia. Austr Systematic Bot 4:299-324

Duke NC, Benzie JAH, Goodall JA, Ballment ER (1998) Genetic structure and evolution of species in the mangrove genus Avicennia (Avicenniaceae) in the Indo-West Pacific. Evolution 52:1612-1626

Everett J (1994) New combinations in the genus Avicennia (Avicenniaceae) Telopea 5:627-629

Excoffier L, Smouse PE, Quattro JM (1992) Analysis of molecular variance inferred from metric distances among DNA haplotypes: application to human mitochondrial DNA restriction data. Genetics 131:479-491

Falk DA (1991) Joining biological and economic models for conserving plant genetic diversity. In: Falk DA, Holsinger KE (eds) Genetics and conservation of rare plants. Oxford University Press, New York, pp 209-224

Fisher MC, Koenig G, White TJ, Taylor JW (2000) A test for concordance between the multilocus genealogies of genes and microsatellites in the pathogenic fungus Coccidioides immitis. Mol Biol Evol 17:1164-1174

Goldstein DB, and Schlötterer C (1999) Microsatellites: evolution and applications. Oxford University Press, Oxford

Huff DR, Peakall R, Smouse PE (1993) RAPD variation within and among natural populations of outcrossing buffalograss [Buchloë dactyloides (Nutt.) Engelm]. Theor Appl Genet 86: 927-934

Hutchings P, Saenger P (1987) Mangrove ecology. Queensland University Press, St Lucia, Australia

Janssen P, Coopman R, Huys G, Swings J, Bleeker M, Vos P, M, Kersters K (1996) Evaluation of AFLP as a new tool in bacterial taxonomy. Microbiology 142:1881-1893

Jarne P, Lagoda PJL (1996) Microsatellites, from molecules to populations and back. Trends Ecol Evol 11:424-429

Jones CJ, Edwards KJ, Castaglione S, Winfield MO, Sala F, van de Wiel C, Bredemeijer G, Vosman B, Matthes M, Daly A, Brettschneider R, Bettini P, Buiatti M, Maestri E, Malcevschi A, Marmiroli N, Aert R, Volckaert G, Rueda J, Linacero R, Vazquez A, Karp A (1997) Reproducibility testing of RAPD, AFLP and SSR markers in plants by a network of European laboratories. Mol Breed 3:381-390

Karp A, Edwards K, Bruford M, Vosman B, Morgante M, Seberg O, Kremer A, Boursot P, Arctander P, Tautz D, Hewitt G (1997) Newer molecular technologies for biodiversity evaluation: opportunities and challenges. Nature Biotechnol 15:625-628

Lynch M, Milligan BG (1994) Analysis of population genetic structure with RAPD markers. Mol Ecol 3:91-99

Maguire TL, Collins GG, Sedgley M (1994) A modified CTAB DNA extraction procedure for plants belonging to the family Proteaceae. Plant Mol Biol Rep 12:106-109

Maguire TL, Edwards KJ, Saenger P, Henry R (2000a) Characterisation and analysis of microsatellite loci in a mangrove species Avicennia marina (Forsk.) Vierh. (Avicenniaceae). Theor Appl Genet 101:279-285

Maguire TL, Saenger P, Baverstock P, Henry R (2000b) Microsatellite analysis of genetic structure in the mangrove species Avicennia marina (Forsk.) Vierh. (Avicenniaceae). Mol Ecol 9:1853-1862

Mantel NA (1967) The detection of disease clustering and a generalised regression approach. Cancer Res 27:209-220

Marshall DR, Brown AHD (1975) Optimum sampling strategies in genetic conservation In: Frankel OH, Hawkes JG (eds) Crop genetic resources for today and tomorrow. Cambridge University Press, London, pp 53-80

Michalakis Y, Excoffier L (1996) A generic estimation of population subdivision using distances between alleles with special reference for microsatellite loci. Genetics 142:1061-1064

Mueller UG, Wolfenbarger LL (1999) AFLP genotyping and fingerprinting. Trends Ecol Evol 14:389-393 
O'Hanlon PC, Peakall R (2000) A review of new PCR-based genetic markers and their utility to weed ecology. Weed Res 40:239-254

Peakall R, Smouse PE (2001) GenAlEx V5: Genetic Analysis in Excel. Population genetic software for teaching and research. Australian National University, Canberra, Australia. http:// www.anu.edu.au/BoZo/GenAlEx/

Peakall R, Gilmore S, Keys W, Morgante M, Rafalski A (1998) Cross-species amplification of soybean (Glycine max) simple sequence repeats (SSRs) within the genus and other legume genera - implications for the transferability of SSRs in plants. Mol Biol Evol 15:1275-1287

Peakall R, Smouse PE, Huff DR (1995) Evolutionary implications of allozyme and RAPD variation in diploid populations of Buffalograss. (Buchloë dactyloides Nutt. Engelm.). Mol Ecol 4:135-147

Pejic I, Ajmone-Marsan P, Morgante M, Kozumplick V, Castiglioni P, Taramino G, Motto M (1998) Comparative analysis of genetic similarity among maize inbred lines detected by RFLPs, RAPDs, SSRs, and AFLPs. Theor Appl Genet 97:1248-1255

Petit RJ, Mousadik AE, Pons O (1998) Identifying populations for conservation on the basis of genetic markers. Conservation Biol 12:844-855

Powell W, Morgante M, Andre C, Hanafey M, Vogel J, Tingey S, Rafalski A (1996) The comparison of RFLP, RAPD, AFLP and SSR (microsatellite) markers for germplasm analysis. Mol Breed 2:225-238

Qureshi TMD (1990) Experimental plantation for rehabilitation of mangrove forest in Pakistan. ISME Mangrove Ecosystems: occasional papers No. 4:1-37
Russell JR, Fuller JD, Macaulay M, Hatz BG, Jahoor A, Powell W, Waugh R (1997) Direct comparisons of levels of genetic variation among barley accessions detected by RFLPs, AFLPs, SSRs and RAPDs. Theor Appl Genet 95:714-722

Saenger P (1998) Mangrove vegetation: an evolutionary perspective. Marine Freshwater Res 49:277-286

Saenger P, Siddiqi NA (1993) Land from the sea: the mangrove afforestation program of Bangladesh. Ocean and Coastal Manag 20:23-39

Schoen DJ, Brown ADH (1993) Conservation of allelic richness in wild crop relative is aided by assessment of genetic markers. Proc Nat Acad Sci 90:10623-10627

Smouse PE, Long JC, Sokal RR (1986) Multiple regression and correlation extensions of the Mantel test of matrix correspondence. Systematic Zool 35:627-632

Smouse PE, Peakall R (1999) Spatial autocorrelation analysis of individual multiallele and multilocus genetic structure. Heredity 82:561-573

Teulat B, Aldam C, Trehin R, Lebrun P, Barker JHA, Arnold GM, Karp A, Baudouin L, Rognon F (2000) An analysis of genetic diversity in coconut (Cocos nucifera) populations from across the geographic range using sequence-tagged microsatellites (SSRs) and AFLPs. Theor Appl Genet 100:764-771

Vos P, Hogers R, Bleeker M, Reijans M, Van der Lee T, Hornes M, Frijters A, Pot J, Peleman J, Kuiper M, Zabeau M (1995) AFLP: a new technique for DNA fingerprinting. Nucleic Acids Rese 23:4407-4414

Zabeau M, Vos P (1993) Selective restriction fragment amplification: a general method for DNA fingerprinting. European Patent Application 92402629.7 (publ. no. 0534858 A1) 